\title{
Comparative Evaluation of Photon Cross-Section Libraries for Materials of Interest in PET Monte Carlo Simulations
}

\author{
Habib Zaidi
}

\begin{abstract}
The many applications of Monte Carlo modeling in nuclear medicine imaging make it desirable to increase the accuracy and computational speed of Monte Carlo codes. The accuracy of Monte Carlo simulations strongly depends on the accuracy in the probability functions and, thus, on the cross-section libraries used for photon-transport calculations. A comparison between different photon cross-section libraries and parameterizations implemented in Monte Carlo simulation packages developed for positron emission tomography and the most recent Evaluated Photon Data Library (EPDL97) developed by the Lawrence Livermore National Laboratory, Livermore, CA, was performed for several human tissues and common detector materials for energies from $1 \mathrm{keV}$ to $1 \mathrm{MeV}$. Different photon cross-section libraries and parameterizations show quite large variations when compared to the EPDL97 coefficients. This latter library is the more up-to-date complete and consistent library available, and was carefully designed in the form of look-up tables providing efficient data storage, access, and management. EPDL97 is already a standard in the nuclear reactor industry. Its use as a standard in the simulation of medical imaging systems will help to eliminate potential differences between the results obtained with different codes. Together with the optimization of the computing time performances of the Monte Carlo software package, Eidolon, photon transport in three-dimensional (3-D) positron emission tomography could be efficiently modeled to develop accurate scatter models and better understand scatter correction techniques.
\end{abstract}

Index Terms-Monte Carlo, Photon cross-section library, Photon transport, positron emission tomography (PET).

\section{INTRODUCTION}

$\mathbf{T}$ HE Monte Carlo method is widely used for solving problems involving statistical processes. In particular, it is employed in the modeling of nuclear medical imaging systems, due to the stochastic nature of radiation emission and transport, and of detection processes. The method is very useful for complex problems that cannot be modeled analytically or when experimental measurements may be impractical. Also, simulation yields "perfect knowledge" of photon histories. In comparison, there is no definitive way to distinguish small-angle-scattered

Manuscript received December 13, 1999; revised June 12, 2000 and September 18, 2000. This work was supported by the Swiss Federal Office for Education and Science under Grant 96.193 within the European Esprit LTR Project PARAPET (EP23493).

The author is with the Division of Nuclear Medicine, Geneva University Hospital, CH-1211 Geneva 14, Switzerland.

Publisher Item Identifier S 0018-9499(00)11047-0. from unscattered events based only on experimental data. As an example, Monte Carlo modeling allows a detailed investigation of the spatial and energy distributions of Compton scatter, which is difficult to measure using present experimental techniques, even with very good energy resolution detectors [1].

The lack of inherent error estimates and relatively slow convergence is a well-known limitation of the Monte Carlo technique. Accurate Monte Carlo simulations rely on detailed understanding and modeling of radiation transport and on the availability of reliable physically consistent databases [2]. As discussed and historically reviewed in some detail by Hubbell [3], there exist many compilations of photon cross-section data. The discrepancies and envelope of uncertainty of available interaction data have been examined from time to time, including the effects of molecular and ionic chemical binding, particularly in the vicinity of absorption edges.

The Lawrence Livermore National Laboratory (LLNL), Livermore, CA, houses the world's most extensive nuclear and atomic cross section database, which parameterizes the interactions of photons, electrons/positrons, neutrons, protons, and other heavy-charged particles. A key feature of the LLNL database is that it is the only exhaustive interaction cross section compilation available. A comparison between an up-to-date source of cross-section data developed by LLNL in collaboration with the National Institute of Standards and Technology (NIST), the Evaluated Photon Data Library (EPDL97) [4] with other more familiar photon interaction databases, XCOM [5] and PHOTX [6], and parameterizations implemented in Monte Carlo packages, GEANT [7] and PETSIM [8] in the interval from 1 to $1000 \mathrm{keV}$ was performed for some human tissues and detector materials of interest in positron emission tomography (PET) imaging.

Although XCOM, PHOTX, and EPDL97 are treated in this paper as independent databases, it is recognized that they are more or less closely related. In particular, XCOM and PHOTX were both produced at NIST and EPDL97 as a result of a long and fruitful collaboration between LLNL and NIST. However, significant differences between the different libraries were reported for low energies [4] and the cross-section data are sensitive to the type of interpolation used for intermediate energies. The EPDL97 library was customized and integrated in our simulation environment significantly improving the efficiency of the Eidolon Monte Carlo simulation package in modeling cylindrical three-dimensional (3-D) positron tomographs [9]. 


\section{Modeling Photon Transport In Monte Carlo Codes}

Many general-purpose Monte Carlo programs have been in use in the field of nuclear imaging and internal dosimetry, with many of them available in the public domain [2], [10]. The EGS4 code [11] represents actually the state of the art of radiation transport simulation in medical radiation physics, since it is very flexible well-documented and extensively tested. The GEANT package [7] was originally designed for high-energy physics experiments, but has found applications also outside this domain in the areas of medical and biological sciences, radiation protection and astronautics. Many other simulation packages developed mainly for nuclear medical imaging research have been described in the literature. This includes codes designed to model projection data for simulated SPECT [12]-[14], PET [9], [15] or both imaging modalities [16]. Some of these codes are based on existing simulation packages mentioned above (EGS4, MCNP, GEANT). A survey of Monte Carlo programs commonly used in nuclear medical imaging and their key features can be found in [2], [10]. Those Monte Carlo software packages are excellent research tools, and many of them could be freely obtained.

The Eidolon Monte Carlo software used in this investigation was developed to simulate cylindrical 3-D positron tomographs. The original code was written in Objective- $\mathrm{C}$ and run under the NextStep object-oriented development environment [9]. The current version of the package can be ported to most of the current hardware platforms and operating systems as far as the platform supports the GNU C compiler (gcc) available from the Free Software Foundation. Boston, MA, which comes with an Objective-C compiler and a runtime library in versions 2.7.1 or newer. This environment was used to develop a parallel implementation of Eidolon where the random seeds are appropriately defined and distributed to different processors such that each processor simulates independent particles histories. Thus, a linear speed-up factor with the number of processors has been achieved [17].

For radiation transport problems, the computational model includes geometry and material specifications. Object modeling is fundamental to perform photon transport efficiently using the Monte Carlo method. It consists of a description of the geometry and material characteristics for the object. The material characteristics of interest are density and energy-dependent cross sections. Different steps are followed when tracing the photon in both the phantom and the detector volume. Annihilation photons are generated within the phantom in or out of the scanner's field-of-view. Both annihilation photons are then tracked independently until a photoelectric interaction occurs in the detector volume or they escape from the geometrical acceptance of the imaging system. The relative ratios of the cross sections for photoelectric effect, incoherent and coherent scattering to the total cross section are used to choose randomly which process occurs at each interaction vertex. The Klein-Nishina expression for the differential cross section per electron for an incoherent interaction is used to sample the energy and polar angle of the incoherently scattered photon taking into account the incoherent scattering factor. The coherent scattering results only in a change in the direction of the scattered photon since the momentum change is transferred to the whole atom. The random number composition and rejection technique is used to sample the momentum of the scattered photon and the scattering angle according to the form-factor distributions. Coherent scatter distributions are sharply forward-peaked and vary considerably with atomic number and energy [18]. The pathlength of the interacting photon is randomly generated according to the exponential attenuation based on the interaction length. The total cross section at the energy of the interacting photon determines the inter-action length of the exponential distribution.

A 3-D PET scanner is simulated as a number of detection rings, each ring consisting of a number of scintillator crystals. The detection block, typical of current generation PET, scanners is simulated by grouping crystals in matrices. Photon history is tracked within a crystal, across crystals within a block and across blocks. Crystals are considered as adjacent in the transaxial plane and in the axial direction. Two external shields are simulated as tungsten rings located at the two axial edges of the tomograph, partly shielding radiation coming from outside the scanner FOV. In the detector blocks, at each interaction vertex, the local energy deposition is recorded. Tracking is stopped either by a photoelectric absorption, escape of the photon from the block volume, or by a Compton scattering leaving less than $5 \mathrm{keV}$ to the recoil photon. The energies of all interaction vertices are summed to yield the total absorbed energy in the scintillation detector. This total energy is assumed to be converted to scintillation light using a Gaussian random smearing to account for the combined energy resolution, $\Delta E / E$, of the scintillator and its photomultipliers. $\Delta E / E$ is assumed to be proportional to $1 / \sqrt{E}$. The position blurring step then calculates the mean detection coordinates $(X, Y, Z)$ of each incident photon. This is done by computing the centroid of all interaction vertices, each weighted by the ratio of its individual energy to the total energy. The mean $X$ and $Y$ coordinates of each photon are smeared to account for spatial resolution degradation due to positron range effect, annihilation photon accolinearity and position miscoding in the detector block.

\section{Photon CRoss-Section LibraRies AND PARAMETRIZATIONS}

The Storm and Israel photon cross-section data [19] have been used extensively in medical physics. This is a 1970 compilation of data for elements $1-100$ and energies $1 \mathrm{keV}-100 \mathrm{MeV}$, and contains mass attenuation coefficients, mass energy-transfer coefficients, and mass-energy absorption coefficients, presented in units of barns/atom. The medical physics community makes extensive use of these coefficients in different applications including Monte Carlo modeling. Table I lists Monte Carlo codes used to simulate nuclear medicine imaging systems together with the corresponding photon cross-section libraries. In the MCNP Monte Carlo code [20], the photon interaction tables for $Z=84,85,87,88,89,91$, and 93 are based on the compilation of Storm and Israel from $1 \mathrm{keV}$ to $15 \mathrm{MeV}$. For all other elements from $Z=1$ through $Z=94$ the photon interaction tables are based on data from Evaluated Nuclear Data Files (ENDF) 
TABLE I

COMmon Monte Carlo Software Packages

Used For Simulating Nuclear MEdical IMAging

SYSTEMS AND CORRESPONDING PHOTON CROSS

SECTION LIBRARIES AND PARAMETERIZATIONS

\begin{tabular}{ll}
\hline Monte Carlo code & Photon cross section library \\
\hline EGS4 [11] & Storm \& Israel [19] \\
& PHOTX [6] \\
ITS including TIGER CYLTRAN and & XCOM [5] \\
ACCEPT [24] & \\
MCNP [20] & Storm \& Israel [19] \\
& ENDF [21] \\
GEANT [7] & EPDL89 [22] \\
SIMSET [16] & GEANT's parametric model [7] \\
SIMIND [12] & PETSIM's Parametric model [8] \\
SIMSPECT [13] & EPDL94 [38], since 1997 [18] \\
& XCOM [5] \\
& Storm \& Israel [19] \\
ENDF [21] \\
MCMATV [14] & EPDL89 [22] \\
PETSIM [15] & NBS [39] and polynomial fitting \\
Triumph's simulator [40] & PETSIM's Parametric model [8] \\
EIDOLON [9,17] & GEANT's parametric model [7] \\
& GEANT's parametric model [7] \\
\hline & EPDL97 [4] since 1999 [37] \\
\hline &
\end{tabular}

[21], [41] from $1 \mathrm{keV}$ to $100 \mathrm{MeV}$. Data above $15 \mathrm{MeV}$ for the Storm and Israel data and above $100 \mathrm{MeV}$ for the ENDF data come from adaptation of the Livermore Evaluated Photon Data Library (EPDL89) [22] and go up to $100 \mathrm{GeV}$. The original EGS4 system [11] also uses compilation by Storm and Israel for the photoelectric and pair production cross sections. Recently, cross-section data for this code, based on the PHOTX library [23] was created by Sakamoto [6]. ITS [24] includes cross sections for bound electrons, the effect of which is ignored in the default EGS4 package. For the photon energy range over 1 $\mathrm{keV}$ to $50 \mathrm{MeV}$, of most interest to medical physicists, particular attention is called to a recently assembled electronic database, including values of energy absorption coefficients, developed by Boone and Chavez [25]. In addition to interaction coefficients, other useful data such as the density, atomic weight, $K, L_{1}, L_{2}, L_{3}, M$, and $N$ edges, and numerous characteristic emission energies are output from the program, depending on a single input variable.

For the energies of interest in nuclear medical imaging (below $1 \mathrm{MeV}$ ), when a photon passes through matter, any of the three interaction processes (photoelectric, incoherent scattering, coherent scattering) may occur. Every cross section depends on the incident particle energy and material composition and on the interaction it undergoes. These partial cross sections are summed to form the total cross section; the ratio of the partial cross section to the total cross section gives the probability for a particular interaction to occur. The probability of a photon with a given energy $E$ to undergo photoelectric absorption or scattering when crossing a layer of material $Z$ can be expressed quantitatively
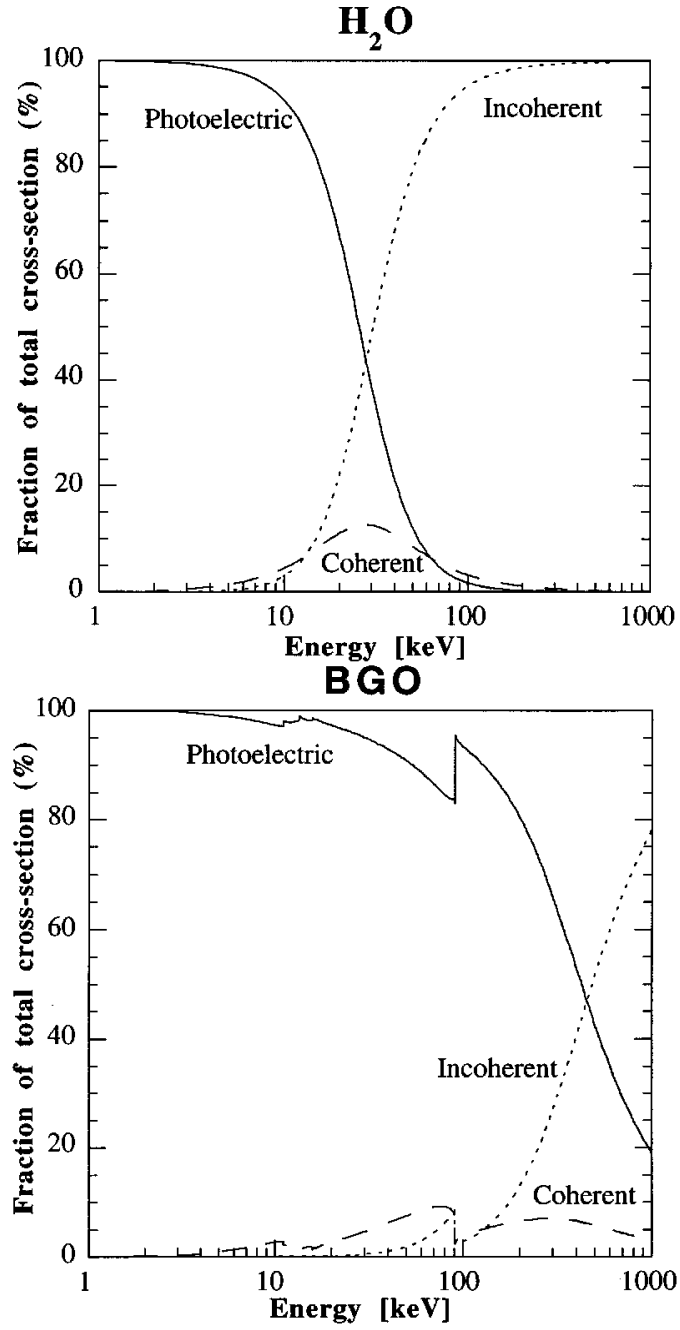

Fig. 1. Components of photon cross sections for water $\left(\mathrm{H}_{2} \mathrm{O}\right)$ and bismuth germanate (BGO) showing the relative contribution and dominance region of each process.

in terms of the linear attenuation coefficients $\mu\left(\mathrm{cm}^{-1}\right)$, which is dependent on the material's density, $\rho\left(\mathrm{g} / \mathrm{cm}^{3}\right)$ :

$$
\mu=\mu_{\text {photo }}+\mu_{\text {incoh }}+\mu_{\text {coh. }}
$$

Many approximations are made in Monte Carlo simulation packages to either simplify the computational model or improve the speed of operation. It is common to neglect coherent scattering in PET Monte Carlo simulation of photon transport because of its low contribution to the total cross section at 511 $\mathrm{keV}$. In order to justify some of the approximations made in Monte Carlo codes, the relative importance of the various processes involved in the energy range below $1 \mathrm{MeV}$ were considered for some compounds and mixtures of interest in nuclear medicine imaging. Fig. 1 illustrates the relative strengths of the photon interactions versus energy for water $\left(\mathrm{H}_{2} \mathrm{O}\right)$ and bismuth germanate (BGO), respectively. For water, a moderately low- $Z$ material, we denote two distinct regions of single interaction dominance: a photoelectric domain below $20 \mathrm{keV}$ and an incoherent scattering domain above $20 \mathrm{keV}$. The coherent scattering contribution to the total cross section is less than $1 \%$ for energies above $250 \mathrm{keV}$. However, this contribution is in the order 
of $7 \%$ for high- $Z$ materials like BGO. Therefore, efforts should be made to treat the coherent scattering process adequately for detector materials.

The most recent EPDL97 attenuation coefficients were compared for some human tissues (water, cortical bone, brain tissue, inflated lung and air) and detector materials ( $\mathrm{NaI}(\mathrm{Tl})$, BGO, BaF2, LSO:Ce and LuAP:Ce) of interest in PET imaging to earlier libraries (PHOTX, XCOM) and parameterizations implemented in Monte Carlo simulation packages (GEANT, PETSIM) over the energy interval from 1 to $1000 \mathrm{keV}$. Appropriate conversions between barns and $\mathrm{cm}^{-1}$ were made for each element. The macroscopic cross section, $\sigma\left(\mathrm{cm}^{-1}\right)$ for a compound or a mixture is given by

$$
\sigma=\rho N_{a} \sum_{i} \frac{w_{i}}{A_{i}} \sigma_{i}
$$

where $N_{a}$ is Avogadro's number $\left(6.0221367 \times 10^{23}\right.$ atoms/mol), $\rho$ the density of the material, $Z_{i}, A_{i}, W_{i}$, and $\sigma_{i}$ are the atomic number, the atomic mass, the fraction by weight, and the atomic cross section of the $i$ th component of the medium, respectively. Human tissue composition data are taken from ICRU 44 report [26].

For libraries where the mass attenuation coefficients $(\mu / \rho)_{i}$ are directly available for individual elements, the linear attenuation coefficients for mixtures and compounds are obtained according to

$$
\mu=\rho \sum_{i} w_{i}(\mu / \rho)_{i} .
$$

\section{A. XCOM}

The compilation of XCOM [5] released on floppy disks for personal computers is available from The National Institute of Standards and Technology (NIST), U.S., through its Office of Standard Reference Data. It generates cross sections and attenuation coefficients for all elements, compounds and mixtures as needed over a wide range of energies. The interpolation procedures used for these tables are slightly different from those used by Berger and Hubbell [5]. A cubic Hermite interpolant for the individual subshell cross sections rather than a cubic spline for the total photoeffect cross section was used, which results in occasional small differences in the vicinity of $M$ - and $N$-shell edges of high- $Z$ elements. To obtain $(\mu / \rho)_{i}$ values at every absorption edge for all constituent elements, interpolation was performed separately for the cross sections indicated in (1), including the photoeffect cross sections for the individual atomic subshells. This program was used to calculate the attenuation coefficients for the selected compounds presented in this paper.

\section{B. PHOTX}

The PHOTX database from the National Bureau of Standards (now NIST), USA, include interaction cross sections and attenuation coefficients for 100 elements covering the energy range $1 \mathrm{keV}-100 \mathrm{GeV}$ [23]. The interactions considered are coherent and incoherent scatterings, photoelectric absorption, and pair production. A separate table gives the photoeffect cross section for each electron shell. A linear interpolation routine was written to calculate the individual component coefficients between tabulated values in order to obtain a continuous set of cross sections spanning energies from 1 to $1000 \mathrm{keV}$. It should be noted that both XCOM and PHOTX compilations were produced at NIST and are closely related. These databases should in principle be identical, with only the packaging and interpolation procedures for intermediate energies being different.

\section{EPDL97}

The EPDL97 library produced by LLNL in collaboration with NIST [4] includes photon interaction data for all elements with atomic number between $Z=1$ and 100 in the energy range over $1 \mathrm{eV}$ to $100 \mathrm{GeV}$, including photoionization, photoexcitation, coherent and incoherent scatterings, and pair and triplet production cross sections. Data files were truncated to match the needs of the diagnostic imaging community, spanning energies from $1 \mathrm{keV}$ to $1 \mathrm{MeV}$ [25]. The EPDL97 data include the effects of form factors and anomalous scattering factors in the incoherent coefficients, with the electron binding energies therefore contributing a noticeable influence on the shape of the incoherent coefficients.

\section{GEANT}

The main applications of the GEANT simulation package developed at CERN [7] are the transport of particles through an experimental setup for the simulation of detector responses and particle trajectories with their graphical representations. Attenuation coefficients are parameterized through different routines within the software package.

1) Photoelectric Absorption: Let $E$ be the incident gamma energy, and $\alpha=E / m c^{2}$. The photoelectric total cross section per atom has been parameterized as

$$
\sigma(Z, \alpha)=\frac{Z^{\beta}}{\alpha^{\gamma}} F(Z, \alpha) .
$$

The parameters $\beta$ and $\gamma$ result from a fit, and $F(Z, \alpha)$ is defined by (5) at the bottom of the page.

The binding energy in the inner shells $E_{i}$ is parameterized as

$$
E_{i}(Z)=Z^{2}\left(a_{i}+b_{i} Z+c_{i} Z^{2}+d_{i} Z^{3}\right)
$$

$$
F(Z, \alpha)= \begin{cases}\left(\begin{array}{ll}
p_{1 K} / Z+p_{2 K} / \alpha+p_{3 K}+p_{4 K} Z+p_{5 K} \alpha+p_{6 K} Z^{2}+p_{7 K} Z \alpha+ \\
p_{8 K} \alpha^{2}+p_{9 K} Z^{3}+p_{10 K} Z^{2} \alpha+p_{11 K} Z / \alpha^{2}+p_{12 K} \alpha^{3}
\end{array}\right) & E>E_{K} \\
p_{1 L 1} / Z+p_{2 L 1} / \alpha+p_{3 L 1} & E_{L 1}<E \leq E_{K} \\
p_{1 L 2} / Z+p_{2 L 2} / \alpha+p_{3 L 2} & E_{L 2}<E \leq E_{L 1} \\
p_{1 M} & 10 \mathrm{keV} \leq E \leq E_{L 2}\end{cases}
$$


$i=K, L_{1}, L_{2}$, and the constants $a_{i}, b_{i}, c_{i}$, and $d_{i}$ are tabulated inside dedicated functions. The fit was made over 301 data points chosen between $5 \leq Z \leq 100$ and $10 \mathrm{keV} \leq E \leq 50$ $\mathrm{MeV}$.

2) Incoherent Scattering: An empirical cross-section formula is used, which reproduces rather well the Compton scattering data down to $10 \mathrm{keV}$

$$
\begin{aligned}
& \sigma(Z, \alpha) \\
& \quad=P_{1}(Z) \frac{\log (1+2 \alpha)}{\alpha} l+\frac{P_{2}(Z)+P_{3}(Z) \alpha+P_{4}(Z) \alpha^{2}}{1+a \alpha+b \alpha^{2}+c \alpha^{3}}
\end{aligned}
$$

where $P_{i}(Z)=Z\left(d_{i}+e_{i} Z+f_{i} Z^{2}\right)$. The fit was made over 511 data points chosen between $1 \leq Z \leq 100$ and $10 \mathrm{keV} \leq E$ $\leq 100 \mathrm{GeV}$.

3) Coherent Scattering: An empirical cross-section formula is used to produce the Rayleigh scattering data

$$
\sigma(Z, E)=a E^{3}+b E^{2}+c E+d
$$

For each element, the fit was obtained over 27 experimental values of the total coherent cross section. The values of the coefficients are stored in a statement within the considered routine.

\section{E. PETSIM}

PETSIM has been developed to model the source distribution and tissue attenuation characteristics, as well as septa and detectors used in PET [15]. In this code, the incoherent scattering linear attenuation coefficients are calculated by multiplying the electron density of the material by the total Compton scattering cross sections, that is [27]:

$$
\begin{aligned}
\sigma_{\text {incoh }}=\rho_{e} 2 \pi r_{o}^{2}\left\{\frac{1+\alpha}{\alpha^{2}}(\right. & \left.\frac{2(1+\alpha)}{1+2 \alpha)}-\frac{\ln (1+2 \alpha)}{\alpha}\right) \\
& \left.+\frac{\ln (1+2 \alpha)}{2 \alpha}-\frac{1+3 \alpha}{(1+2 \alpha)^{2}}\right\}
\end{aligned}
$$

where $r_{o}$ is the classical electron radius and $\rho_{e}$ the electron density of the material (in electrons $/ \mathrm{cm}^{3}$ ) calculated using the relationship:

$$
\rho_{e}=\rho N_{a} \sum_{i} \frac{w_{i} Z_{i}}{A_{i}}
$$

A linear log-log fitting was performed using the XCOM database on each side of the absorption edge to compute the 4 parameters required to calculate the photoelectric linear attenuation coefficient, which is assumed to have the following form [8]:

$$
\tau= \begin{cases}A E^{-B_{1}}, & E>E_{k} \\ A E^{-B_{2}}, & E<E_{k} .\end{cases}
$$

A similar approach was undertaken to compute relevant parameters for some materials studied in this work and not available in [8].

\section{Phantom Simulations}

Due to different implementations of the software packages described in Section II, it is expected that the output of simula- tion results for the same geometry will be different. To isolate the effect of the photon cross-section library from other software implementation aspects, the Eidolon simulation package [9] was used and the cross-section library changed each time to generate projection data. Evaluation of the effect of the photon cross-section libraries on actual simulation of PET tomographs was performed by generating data sets for a uniformly filled cylinder and a line source centered in a water-filled $20 \mathrm{~cm}$ right circular cylinder to calculate scatter fractions. The Monte Carlo calculations were carried out in ten batches of 10000000 histories and the mean and standard deviation of the ten generated data sets evaluated. It is worth to point out that there are alternative methods that are more computationally efficient. If no variance reduction is used, variance estimates can be obtained from Poisson statistics. Otherwise, this estimate can be made from tallies of squares of the weights as well as of the weights themselves. However, such approximations will underestimate the precision in the estimates of the differences between the results of two runs when the runs are correlated [2]. Simulations were also carried out for the 3-D Hoffman brain phantom [28] contained in a cylindrical homogeneous water phantom $(20 \mathrm{~cm}$ diameter, $17.5 \mathrm{~cm}$ height) with apparent relative concentrations of 4,1 and 0 for gray matter, white matter and the ventricles, respectively, simulating the activity distributions found in normal human brain for cerebral blood flow and metabolism studies currently employed in PET. Phantom data sets were generated for the ECAT 953B PET scanner operated in 3-D mode (16 rings of 384 detectors each with a ring radius of $38 \mathrm{~cm}$ ) with $23 \%$ energy resolution and 4-mm intrinsic spatial resolution [29].

The scatter fraction is of great importance for quantitative estimation of the scattering contribution. It is defined as the ratio between the number of scattered photons and the total number of photons (scattered and unscattered). The Monte Carlo history of each coincidence event was used to evaluate the scatter fraction. This was achieved by counting the detected coincidences for which at least one photon has scattered in the phantom. For the experimentally measured data, the scatter fraction is estimated by extrapolation of the scatter tails using an exponential fit. For the simulated data, the scatter fraction evaluated using the coincidence events' Monte Carlo history agrees with that from the exponential fit within an absolute uncertainty of 3\%. Quantitative evaluation of the difference between results of Monte Carlo simulated scatter fractions was performed using statistical analysis. A two-sample problem in the case of matched and correlated samples was used [30]. The student's t-test values and the corresponding significance levels associated to the student's analysis (one-tailed test, five degrees of freedom), were calculated for the different photon cross-section libraries as compared to EPDL97. If the calculated t-value is greater than the critical $\mathrm{t}$-value, the null hypothesis of no statistically significant difference (in case of a 95\% confidence interval) is rejected. It is worth to point out that failure to prove statistically significant differences is not sufficient to confirm that the results are statistically identical.

Fig. 7 illustrates scatter profiles for a uniform cylindrical phantom for LLD settings of $380 \mathrm{keV}$ and $450 \mathrm{keV}$. Raising or lowering the LLD setting changes the shape of the scatter profile. Nevertheless, the FWHM of the scatter response 


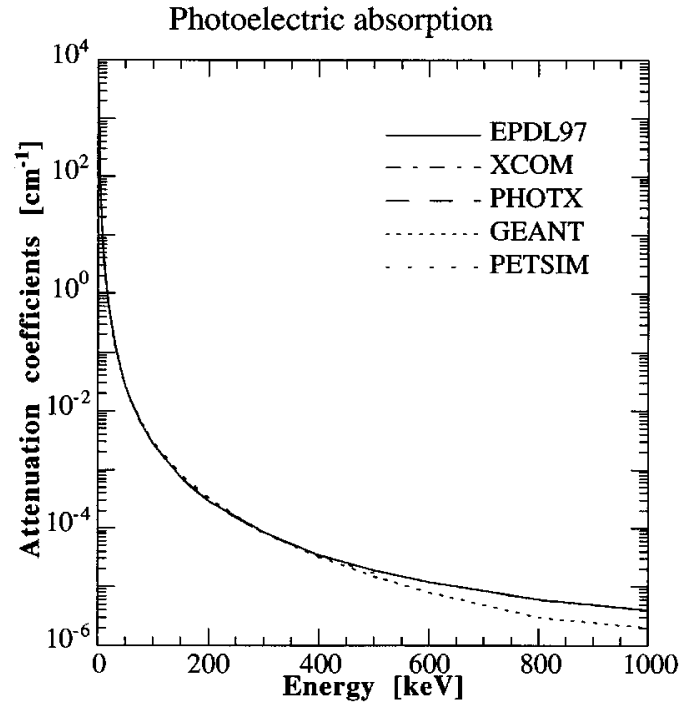

(a)

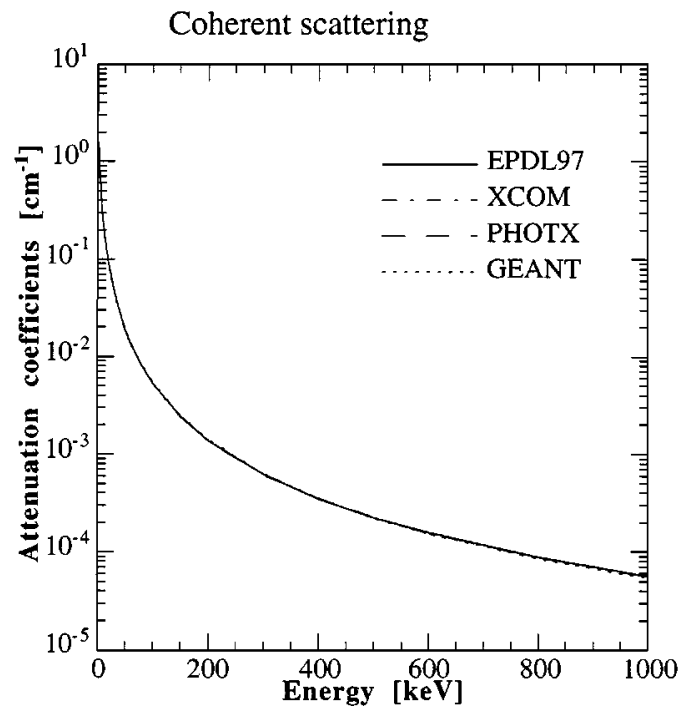

(c)

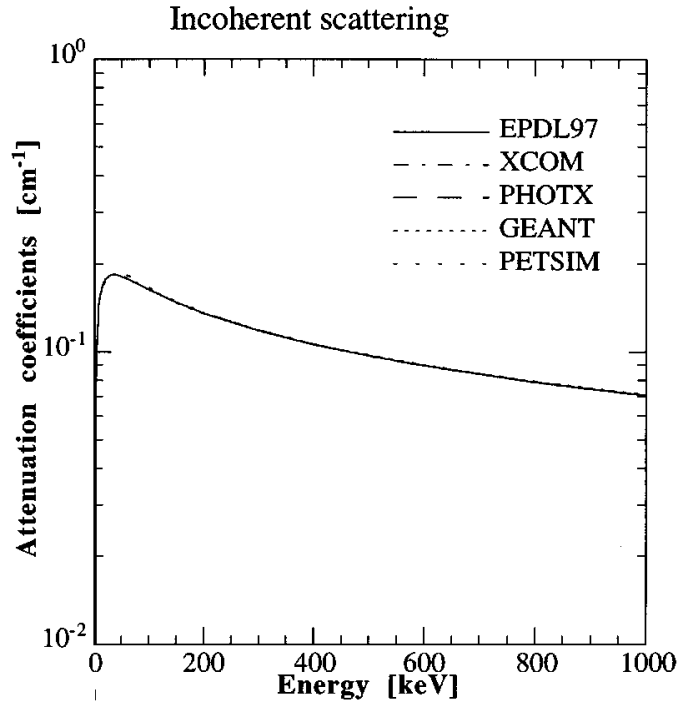

(b)

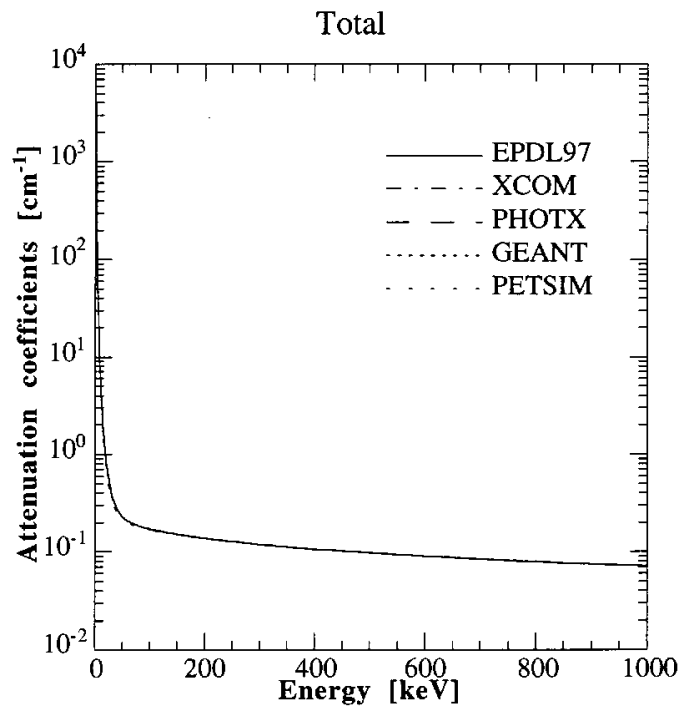

(d)

Fig. 2. Total and partial linear attenuation coefficients versus photon energy for water $\left(\mathrm{H}_{2} \mathrm{O}\right)$ computed using different photon cross-section libraries and parameterizations plotted on a log scale. The different processes: (a) photoelectric absorption, (b) incoherent scattering and (c) coherent scattering contributing to (d) the total linear attenuation coefficient (bottom right) are shown. The coefficients appear to be qualitatively similar in this display format.

function is still significant even when the LLD is very high. The LLD setting affects the accuracy of a correction method based on estimating the scatter profile from the scatter 'tails.' For example, LLD settings of $380 \mathrm{keV}$ place the energy threshold at the lower-energy tail of the photopeak for BGO detector blocks with $23 \%$ energy resolution. A $511 \mathrm{keV}$ photon can undergo Compton scatter with an angle as large as $30^{\circ}$ and still possess $450 \mathrm{keV}$ of energy. XCOM and PETSIM slightly underestimate scatter components for both LLD settings as compared to EPDL97. It has been shown that using different libraries can lead to differences in the scatter distributions of approximately $\pm 8 \%$. It is worth to point out that lowering the LLD setting leads to more appreciable differences between the scatter profiles generated using EPDL97 and the other libraries. Table IV summarizes the statistical comparison between scatter profiles of the uniform cylinder generated using the different libraries as compared to EPDL97. These results further confirm the existence of a statistically significant difference between the resulting projections generated using different libraries $(p<0.025)$.

Comparisons between the profiles through a simulated 2-D projection of the 3-D brain phantom estimated using different libraries before and after applying scatter correction are illustrated in Fig. 8(a) and (b). There is a clear overestimation in the activity level due to contribution from scattered events and the distribution is not uniform.

Scatter and attenuation corrections were applied to the projection data prior to reconstruction. The convolution-subtraction method was used for scatter correction [31]. In this method, the scatter distribution is estimated by iteratively convolving the acquired projections with a mono-exponential scatter kernel $(s r f)$. The scatter estimate $\left(p_{s}\right)$ is then subtracted from the measured data $\left(p_{o}\right)$ after scaling by an appropriate scatter fraction $(S F)$. The observed data are used as a first approximation to the unscattered distribution $\left(p_{u}\right)$ and the process is repeated iteratively with each step using 


\section{$\mathrm{H}_{2} \mathrm{O}$}

\begin{tabular}{|llll|}
\hline$\circ$ & PHOTX & $\square$ & GEANT \\
$\wedge$ & XCOM & + & PETSIM \\
\hline
\end{tabular}

Total

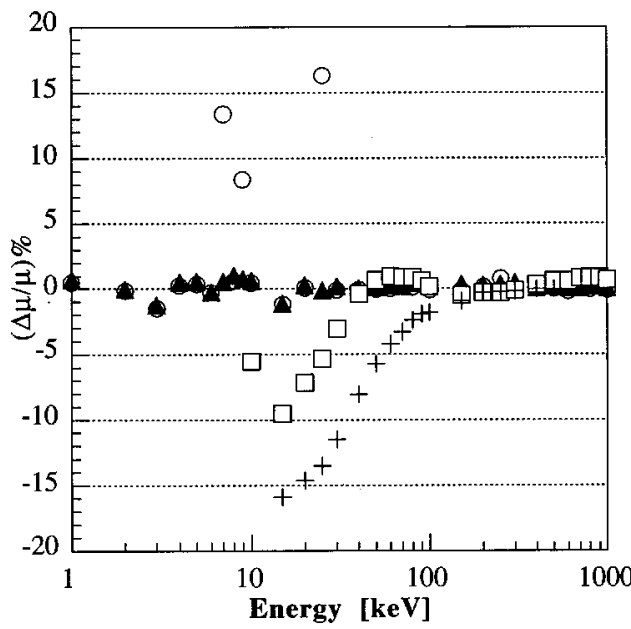

(a)

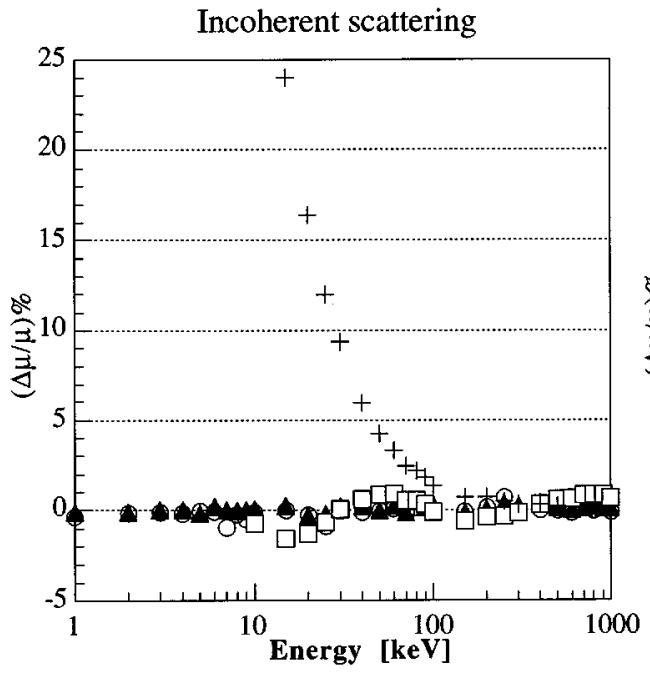

(c)
Photoelectric absorption

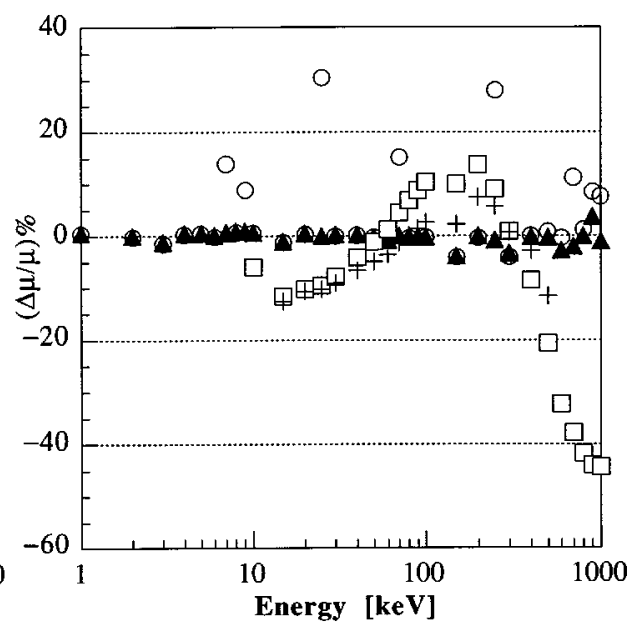

(b)

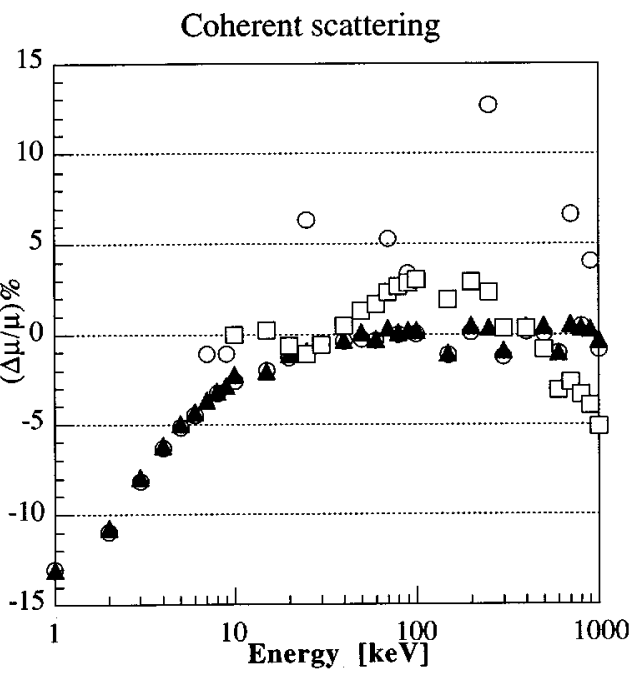

(d)

Fig. 3. Comparisons (percent differences) between the different libraries and the EPDL97 database for water $\left(\mathrm{H}_{2} \mathrm{O}\right)$. The coefficients shown are: (a) total, (b) photoelectric, (c) incoherent, and (d) coherent. The comparisons were calculated at energies given to the keV resolution between 1 and $1000 \mathrm{keV}$ for both $\mathrm{XCOM}$ and PHOTX, between 10 and $1000 \mathrm{keV}$ for GEANT, and between 15 and $511 \mathrm{keV}$ for PETSIM.

the previous estimate of the scatter-free distribution as input to the scatter estimation:

$$
\hat{p}_{u}^{(n)}=p_{o}-S F\left(\hat{p}_{u}^{(n-1)} \otimes s r f\right)
$$

where the " ${ }^{\prime \prime}$ indicates that the parameter is an estimate of the scatter and $n$ is the iteration number. The scatter fraction used was 0.30 and the number of iterations was four. In this work, a simple parameterization of the scatter response function is performed by fitting simulated response functions to a line source in a uniform water-filled cylindrical phantom with mono-exponential kernels [32]. Attenuation correction files were created by forward projecting the 3-D density map estimated with a constant linear attenuation coefficient of $0.096 \mathrm{~cm}^{-1}$. Generated data sets were reconstructed using the reprojection algorithm [33] with a maximum ring index difference of eleven.

\section{RESULTS}

To illustrate roughly the differences between the cross-section libraries, linear attenuation coefficients for water are plotted in Fig. 2 as a function of energy, along with the individual contributions from photoelectric absorption, incoherent scatter and coherent scatter. EPDL97 data points are given with $1 \mathrm{keV}$ steps. 


\section{$\operatorname{NaI}(\mathrm{Tl})$}

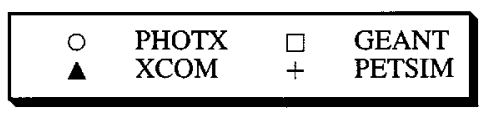

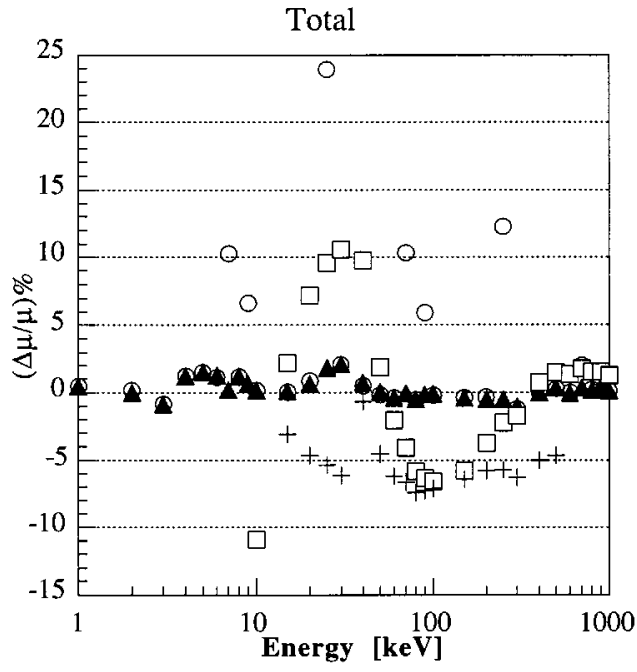

(a)

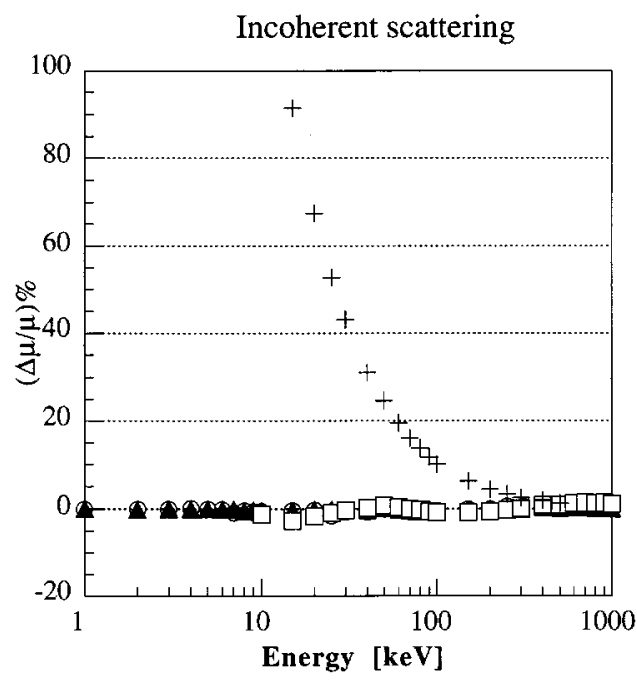

(c)

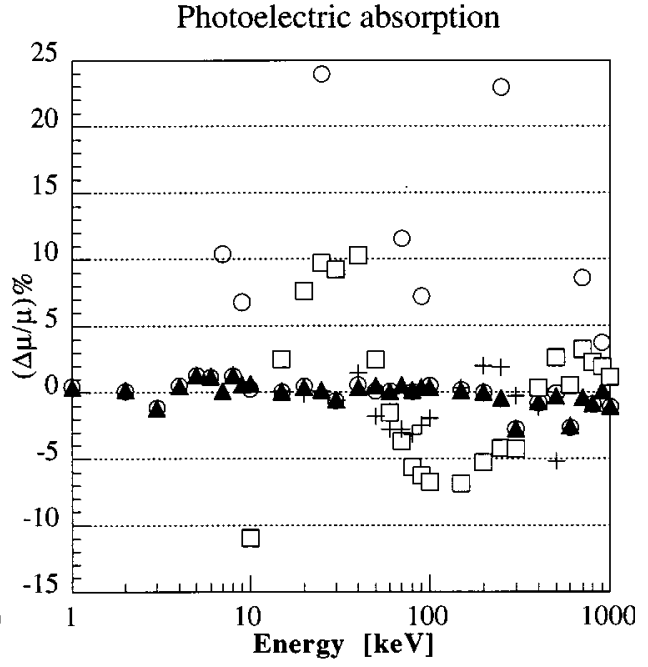

(b)

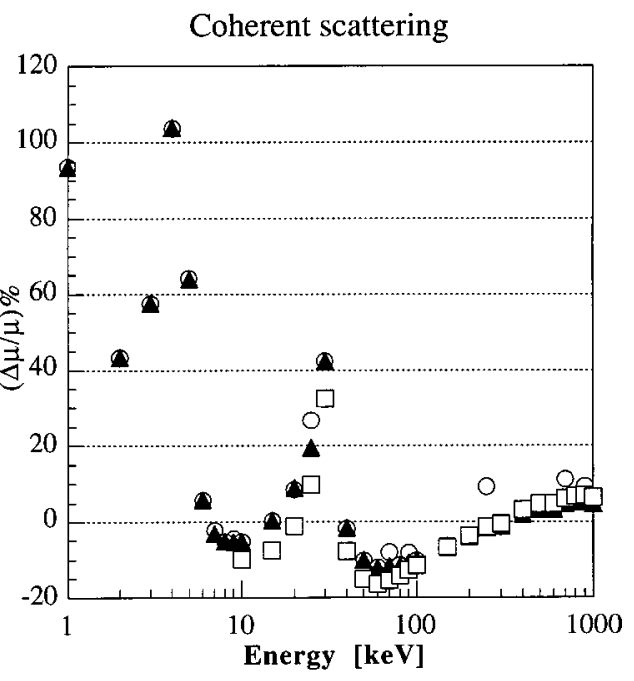

(d)

Fig. 4. Comparisons (percent differences) between the different libraries and the EPDL97 database for sodium iodide (NaI(Tl)). The coefficients shown are: (a) total, (b) photoelectric, (c) incoherent, and (d) coherent. The comparisons were calculated at energies given to the keV resolution between 1 and $1000 \mathrm{keV}$ for both XCOM and PHOTX, between 10 and $1000 \mathrm{keV}$ for GEANT, and between 15 and $511 \mathrm{keV}$ for PETSIM.

The corresponding coefficients in different libraries appear to be qualitatively similar in this display format.

In order to investigate differences between the EPDL97 database and the other libraries and parameterizations, percent differences between the individual coefficients are shown in Figs. 3 and 4 for water and $\mathrm{NaI}(\mathrm{Tl})$, respectively. The percent difference was calculated as $100 \times\left(\mu_{c}-\mu_{\text {EPDL97 }}\right) / \mu_{\text {EPDL97 }}$, where $\mu_{c}$ is the coefficient being compared and $\mu_{\text {EPDL97 }}$ are the EPDL97 coefficients. The comparisons shown in Figs. 3 and 4 are continuous and were calculated at $1 \mathrm{keV}$ intervals between 1 and $1000 \mathrm{keV}$ for both XCOM and PHOTX, between 10 and $1000 \mathrm{keV}$ for GEANT, and between 15 and 511 $\mathrm{keV}$ for PETSIM. It can be seen that the differences can be as large as $20 \%$. For PHOTX, the strong variations and oscillations visible in the plots as high frequency quasiperiodic noise are thought to be due to the discrete nature of the source data and the crude interpolation used between the known coefficients which was chosen mainly to mimic the standard method used by most Monte Carlo developers. The parametric model used in PETSIM has an apparent problem in modeling the incoherent coefficients for low energies $(<100 \mathrm{keV})$. This can be explained by the fact that XCOM uses a combination of the Klein-Nishina formula and nonrelativistic Hartree-Fock incoherent scattering functions (which is about equal to 1 above $100 \mathrm{keV}$ ) to calculate the incoherent scattering, whereas only the Klein-Nishina relationship is used in PETSIM [8]. The dis- 
TABLE II

Global Estimates of Relative Percent Differences Between PHOTX and XCOM Libraries and EPDl97 CoefFicients. Mean Relative ERrors with Standard Deviations Over the EnERgy Range 250 to 511 keV Are Shown

\begin{tabular}{|c|c|c|c|c|c|c|c|c|c|c|c|c|c|c|c|}
\hline & \multicolumn{4}{|c|}{$\mathrm{XCOM}$} & \multicolumn{4}{|c|}{ PHOTX } & \multicolumn{4}{|c|}{ GEANT } & \multicolumn{3}{|c|}{ PETSIM } \\
\hline & Total & Photoelectric & Incoherent & Coherent & Total & Photoelectric & Incoherent & Coherent & Total & Photoelectric & Incoherent & Coherent & Total & Photoelectric & Incolerent \\
\hline Water & $0.24 \pm 0.17$ & $1.74 \pm 0.78$ & $0.24 \pm 0.18$ & $0.40 \pm 0.29$ & $0.28 \pm 0.22$ & $7.67 \pm 7.29$ & $0.26 \pm 0.20$ & $3.74=3.20$ & $0.30=0.13$ & $9.61 \neq 6.82$ & $0.31 \neq 0.13$ & $0.85 \pm 0.44$ & $0.11 \pm 0.08$ & $5.12 \pm 3.79$ & $0.29 \pm 0.11$ \\
\hline Bone (Cort) & $0.21 \pm 0.16$ & $1.40 \pm 0.58$ & $0.20 \pm 0.15$ & $0.29 \pm 0.17$ & $0.59 \pm 0.29$ & $7.53 \pm 7.28$ & $0.50 \pm 0.19$ & $4.0 \pm 3.08$ & $0.32 \pm 0.16$ & $3.10 \pm 1.57$ & $0.33 \pm 0.16$ & $1.65 \pm 0.80$ & $0.42 \pm 0.17$ & $4.21 \pm 3.15$ & $0.50 \pm 0.14$ \\
\hline Lung (Infl) & $0.23 \pm 0.18$ & $1.72 \pm 0.74$ & $0.23 \pm 0.18$ & $0.42 \pm 0.29$ & $0.63 \pm 0.22$ & $7.09 \pm 7.37$ & $0.61 \pm 0.20$ & $4.07 \pm 3.09$ & $0.39 \pm 0.20$ & $8.37 \pm 5.93$ & $0.39 \pm 0.20$ & $0.86 \pm 0.48$ & $0.25 \pm 0.08$ & $4.71 \pm 2.75$ & $0.14 \pm 0.11$ \\
\hline Brain tissue & $0.23 \pm 0.17$ & $4.28 \pm 0.69$ & $0.24 \pm 0.18$ & $0.75 \pm 0.36$ & $0.64 \pm 0.22$ & $7.73 \pm 7.58$ & $0.61 \pm 0.20$ & $3.87 \pm 3.22$ & $0.39 \pm 0.20$ & $8.18 \pm 5.81$ & $0.40 \pm 0.20$ & $0.86 \pm 0.49$ & $0.73 \pm 0.08$ & $4.91 \pm 3.34$ & $0.34 \pm 0.11$ \\
\hline Air & $0.29 \pm 0.21$ & $1.58 \pm 0.68$ & $0.29 \pm 0.21$ & $0.33 \pm 0.24$ & $0.60 \pm 0.38$ & $7.83 \pm 7.66$ & $0.73 \pm 0.31$ & $4.15 \pm 3.22$ & $0.33 \pm 0.24$ & $9.73 \pm 6.90$ & $0.33 \pm 0.24$ & $1.24 \pm 0.97$ & $0.24 \pm 0.16$ & $4.87 \pm 3.43$ & $0.34 \pm 0.22$ \\
\hline $\operatorname{NaI}(\mathrm{Tl})$ & $0.44 \pm 0.39$ & $1.44 \pm 0.70$ & $0.12 \pm 0.07$ & $1.58=0.79$ & $2.83 \pm 3.07$ & $6.37 \pm 6.11$ & $0.32 \pm 0.12$ & $4.85 \pm 2.06$ & $0.98=0.68$ & $1.73 \pm 1.58$ & $0.51 \pm 0.33$ & $2.36 \pm 1.33$ & $5.41 \pm 0.46$ & $2.29 \pm 1.99$ & $1.96 \pm 0.57$ \\
\hline $\mathrm{BGO}$ & $0.73 \pm 0.45$ & $1.30 \pm 0.56$ & $0.11 \pm 0.08$ & $2.25 \pm 1.32$ & $3.52 \pm 3.46$ & $5.01 \pm 4.58$ & $0.22 \pm 0.11$ & $3.83 \pm 1.50$ & $1.01 \pm 0.72$ & $1.96 \pm 1.02$ & $1.60 \pm 0.46$ & $5.03 \pm 2.68$ & $1.92 \pm 0.78$ & $1.76 \pm 1.31$ & $13.94 \pm 0.72$ \\
\hline $\mathrm{BaF}_{2}$ & $0.42 \pm 0.35$ & $1.33 \pm 0.61$ & $0.12 \pm 0.06$ & $1.56 \pm 0.89$ & $2.92 \pm 3.15$ & $6.32 \pm 6.03$ & $0.32 \pm 0.12$ & $4.78 \pm 1.94$ & $0.95 \pm 0.71$ & $1.75 \pm 1.51$ & $0.51 \pm 0.32$ & $3.32 \pm 1.77$ & $5.03 \pm 0.35$ & $2.28 \pm 1.73$ & $2.20 \pm 0.55$ \\
\hline LSO:Ce & $0.50 \pm 0.32$ & $0.91 \pm 0.44$ & $0.13 \pm 0.08$ & $1.98 \pm 1.16$ & $5.14=3.92$ & $7.68 \pm 5.85$ & $1.54 \pm 0.13$ & $5.50 \pm 1.90$ & $1.33 \pm 0.78$ & $1.38 \pm 0.96$ & $1.81 \pm 0.47$ & $5.09 \pm 2.45$ & $3.40 \pm 0.36$ & $1.83 \pm 1.42$ & $7.46 \pm 0.61$ \\
\hline LuAP:Ce & $0.43 \pm 0.29$ & $0.83 \pm 0.42$ & $0.09 \pm 0.06$ & $1.98 \pm 1.16$ & $4.96 \pm 3.85$ & $7.69 \pm 5.89$ & $1.44 \pm 0.12$ & $5.45 \pm 1.89$ & $1.30 \pm 0.76$ & $1.38 \pm 0.96$ & $1.74=0.45$ & $5.06 \pm 2.43$ & $9.24 \pm 1.02$ & $1.83 \pm 1.37$ & $5.66 \pm 0.50$ \\
\hline
\end{tabular}

crepancy is even greater for high atomic number materials since the electrons can no longer be considered free. However, this should not affect the accuracy of photon transport simulations within the phantoms since the low energy cutoff is generally higher than $200 \mathrm{keV}$. But, it might have implications in multispectral imaging where acquisitions in lower energy windows are simulated and used for example in scatter correction based on a multienergy window setting.

The average positive difference between the GEANT and the EPDL97 coefficients for water are $0.3 \%, 9.61 \%, 0.31 \%$, and $0.85 \%$, for the total, photoelectric, incoherent and coherent attenuation coefficients, respectively. Larger differences can be seen near the $K$ or $L$ edges for some of the compounds. A good agreement is observed between EPDL97 and XCOM for all compounds except the low energies region for the coherent attenuation coefficients. With some exceptions, most of the data points fall within the $\pm 3 \%$ region. The mean relative errors for water linear attenuation coefficients in the energy range over 250 to $511 \mathrm{keV}$ between XCOM and EPDL97 are $(1.74 \pm 0.78) \%,(0.24 \pm 0.18) \%,(0.40 \pm 0.29) \%$ and $(0.24 \pm 0.17) \%$ for photoelectric effect, incoherent scattering, coherent scattering and total linear attenuation coefficients, respectively. They are $(5.12 \pm 3.79) \%,(0.29 \pm 0.11) \%$ and $(0.11 \pm 0.08) \%$ between PETSIM and EPDL97 for photoelectric effect, incoherent scattering and total linear attenuation coefficients, respectively. While the overall average difference was small, there was a larger difference $(>50 \%)$ between cross sections for some compounds. The overall percent differences are summarized in Table II for the different materials investigated.

Fig. 5 shows comparisons between measured [29] and simulated [9] scatter fractions estimated using the EPDL97 photon cross-section library as a function of the lower level discriminator (LLD). Discrepancies observed in quantification of this parameter could be explained by the sharp energy threshold model used in our code, which do not consider the nonproportionality of the scintillation response of block detectors (the experimentally observed variations in crystal energy response within a single block is not taken into account in the analysis of simulated data), and the statistical noise from photoelectron amplification in the photomultiplier tubes. A similar effect was reported by Michel et al. [34], where they found that scatter fractions are overestimated (underestimated) below (above) 300 $\mathrm{keV}$. Another argument is that the processing required to analyze the experimental data affects the accuracy of the technique. Moreover, the uncertainties associated with the LLD setting for the measured data, which most likely is lower than the assumed one [35] cannot be easily quantified. Above $500 \mathrm{keV}$, the simulated sensitivity is so low that estimation of scatter fraction values could not be relevant. The ratios of scatter fractions estimated using the different cross-section libraries to those estimated using the EPDL97 library are shown in Fig. 6. There is little difference between estimated scatter fractions. The effect of the cross-section library can hardly be observed when calculating this quantity, which involves only the resulting numbers of unscattered and scattered events. However, it can be seen that PETSIM slightly underestimates the scatter fraction as compared to other libraries. The statistical comparison between scatter fractions estimated using the different libraries as compared to those obtained using EPDL97 is presented in Table III. According to student's t-test, there is a statistically significant difference between the scatter fractions estimated using EPDL97 and the other libraries $(p<0.05)$, except PHOTX $(p \gg 0.20)$.

An example of reconstructed images is shown in Fig. 9 in a comparison of the images of the 3-D brain phantom corrected for scatter using the convolution-subtraction method. About 53 million events were collected for each of the computational models. Profiles through the images are also shown. Results using this approach for scatter correction in cerebral 3-D PET scans (homogeneous attenuating region) have proven to be accurate [31]. Although the data presented here were obtained from low count studies, there is a clear and statistically significant difference $(p<0.025)$ in both projection data and resulting profiles on reconstructed images when using different libraries for generating data sets. For the later case, evaluation was performed on reconstructed scattered projections corresponding to events known to be scattered from 


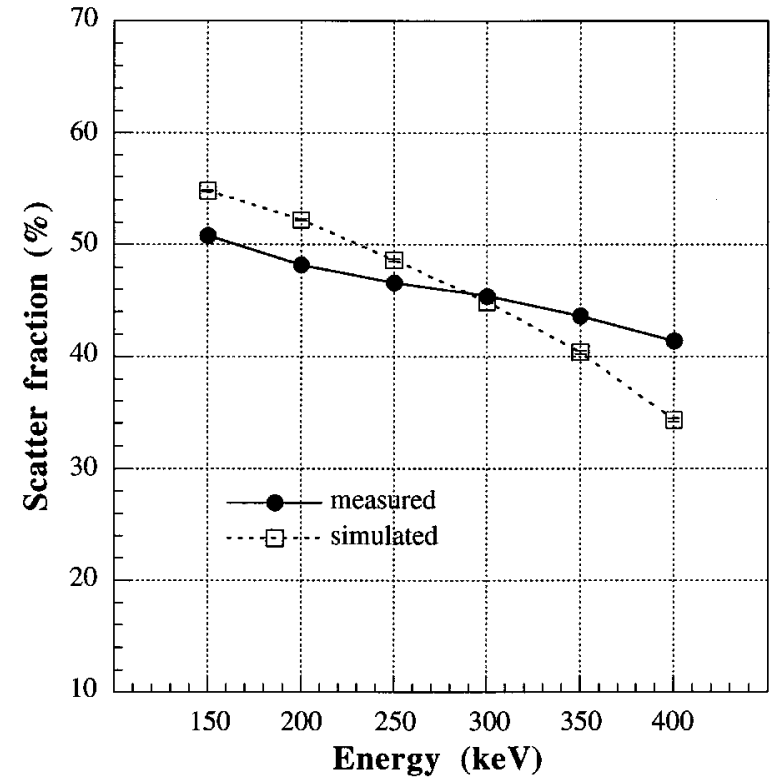

Fig. 5. Comparison between measured and simulated scatter fractions (using the EPDL97 photon cross-section library) in the ECAT 953B PET scanner as a function of the lower level energy discriminator.

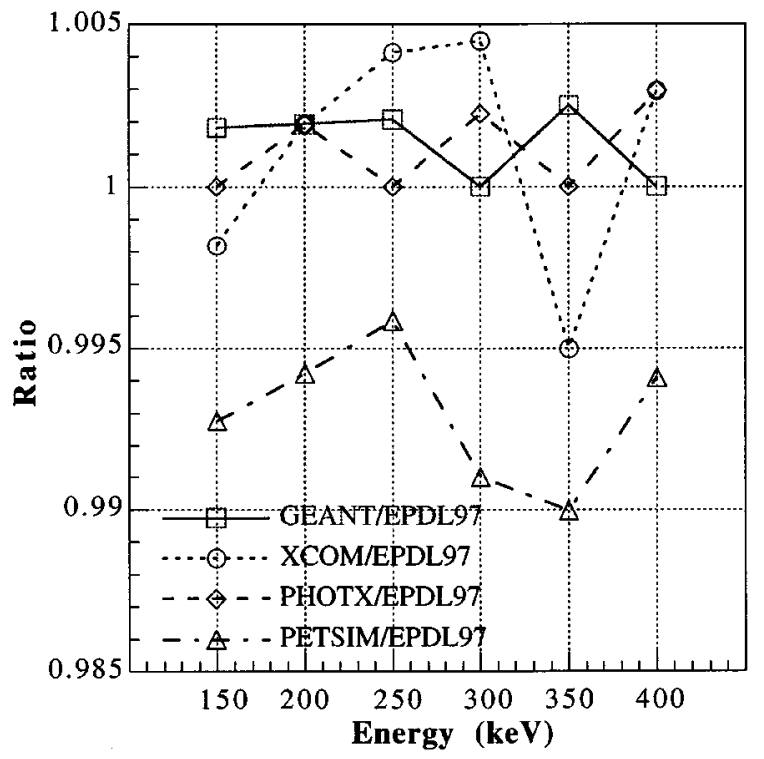

Fig. 6. Ratios between scatter fractions estimated using the different photon cross-section libraries and those estimated using the EPDL97 library for the ECAT 953B PET scanner as a function of the lower level energy discriminator. Error bars are shown on the plots.

the Monte Carlo particle histories to avoid dependence on a particular scatter correction technique (data not shown). This might strongly influence the parameters derived for modeling the scatter response function and the conclusions drawn when evaluating scatter correction techniques using Monte Carlo simulated source distributions [32].

\section{DISCUSSION AND CONCLUSION}

Monte Carlo modeling has contributed to a better understanding of the physics of photon transport in medical physics. The large number of applications of the Monte Carlo method attests to its usefulness as a research tool in different areas of nuclear medicine imaging including detector modeling and systems design, image reconstruction and scatter correction techniques, internal dosimetry and pharmacokinetic modeling [2]. Monte Carlo simulation is a gold standard for the simulation of PET data and is an indispensable tool to develop and evaluate scatter models [9], [32]. The recent interest in the development of high-resolution PET scanners and multiple-processor parallel processing systems has created new opportunities for Monte Carlo simulation in PET imaging [2], [10]. This motivates the question: what is the ultimate accuracy that can be achieved using the Monte Carlo method? The accuracy of simulations is limited by several factors such as accuracy in modeling of annihilation photon noncollinearity, positron range, the photon transport model and associated physical databases used, and the obvious complexity in modeling the human body. Because the overall accuracy is a combination of all these components, when designing a Monte Carlo package that simulates a PET imaging system, it is quite important to understand the effect of each component.

A detailed investigation of the error committed for scanner input parameters on the accuracy of the simulated data, particularly the energy and spatial resolution of the tomograph was not helpful in drawing a general conclusion regarding the sensitivity to a particular parameter. That is, in the absence of an accurate model of the variations of the crystal energy response within a single block [36], changing the energy resolution parameter alone revealed small variations of the scatter fraction similarly to the results reported here when changing the photon cross-section library. Determination of the sources of systematic errors is not an easy task.

Data on the scattering and absorption of photons are fundamental for all Monte Carlo calculations since the accuracy of the simulation depends on the accuracy in the probability functions, i.e. the cross-section data. An aspect that deserves attention is the fact that there exist differences between the physical cross-section data used in available Monte Carlo codes. This is of special importance when comparing results from different codes. The use of different cross-section data and approximations will usually yield different results and the accuracy of the results is generally hard to quantify. It is assumed that the program, which best simulates the true physics and geometry will give the best answer. Given the results presented in this paper, it is expected that photon transport in nonuniformly attenuating medium will exhibit noticeable differences when using different cross-section libraries. Therefore, the reported errors should have a significant effect on most, if not all realistic simulations of nuclear medicine applications. In the first version of the Eidolon public domain simulation package [9], interaction cross sections and scattering distributions were computed from parameterizations implemented in the GEANT simulation package [7]. It has been demonstrated in this work that different photon cross-section libraries show quite large variations as compared to the most recent EPDL97 nuclear data files. Both XCOM and PHOTX use form-factor corrections for incoherent cross sections, but do not use anomalous scattering factors, so their low energy coherent cross sections is orders of magnitude too large. GEANT [7], Eidolon [9], and SIMSET 
TABLE III

Summary of Statistical COMPARISON BetweEn SimUlated SCATter Fractions Estimated Using the EPDL97 LibRARY AND OtheR PHOTON Cross-Section Libraries. The Student's t-Values and Associated Statistical DifFerence Are Shown. The Critical t-Value $\left(t_{\text {crit }}=2.015\right)$ Is Related to a Probability of 0.05 FOR THE Null Hypothesis to OcCuR (“The Two Distributions ARe Not the SAME”)

\begin{tabular}{lcc}
\hline Photon cross section library & Student's t-value & Probability \\
\hline GEANT & 3.0686 & $<0.05$ \\
XCOM & 3.0094 & $<0.05$ \\
PHOTX & 1.3522 & $>>0.20$ \\
PETSIM & 3.9616 & $<0.025$ \\
\hline
\end{tabular}

\section{$\mathrm{LLD}=380 \mathrm{keV}$}
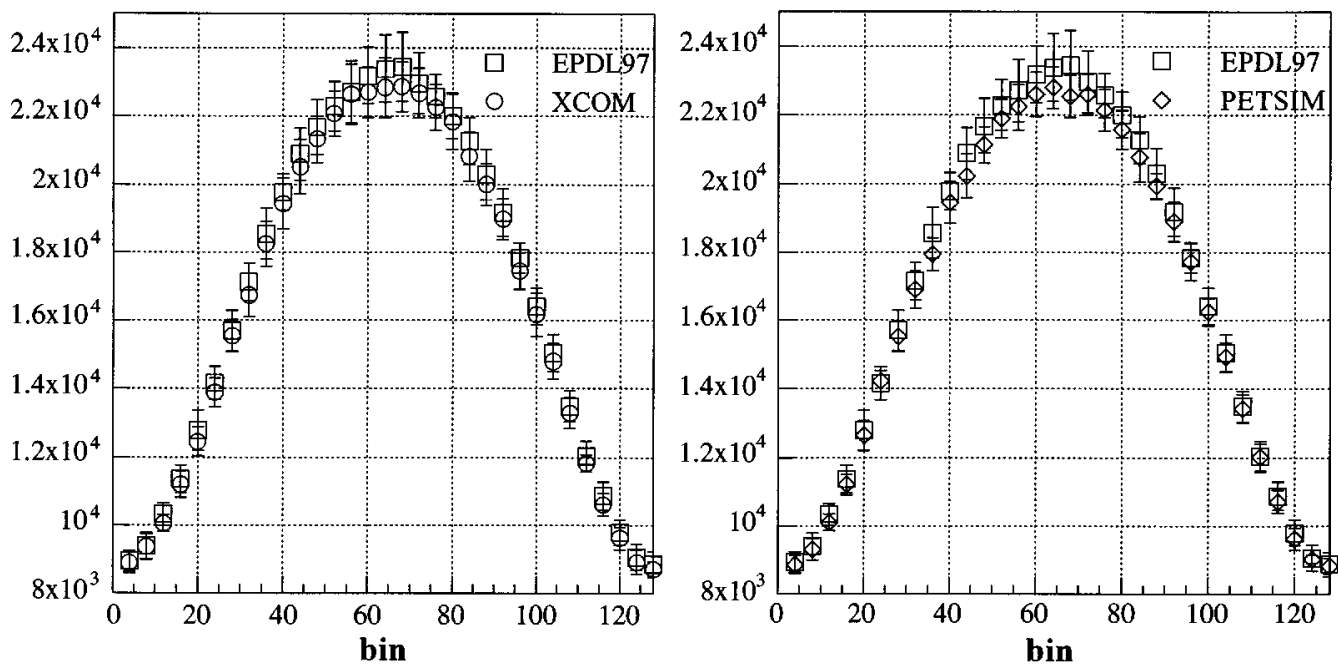

(a)

$\mathrm{LLD}=450 \mathrm{keV}$
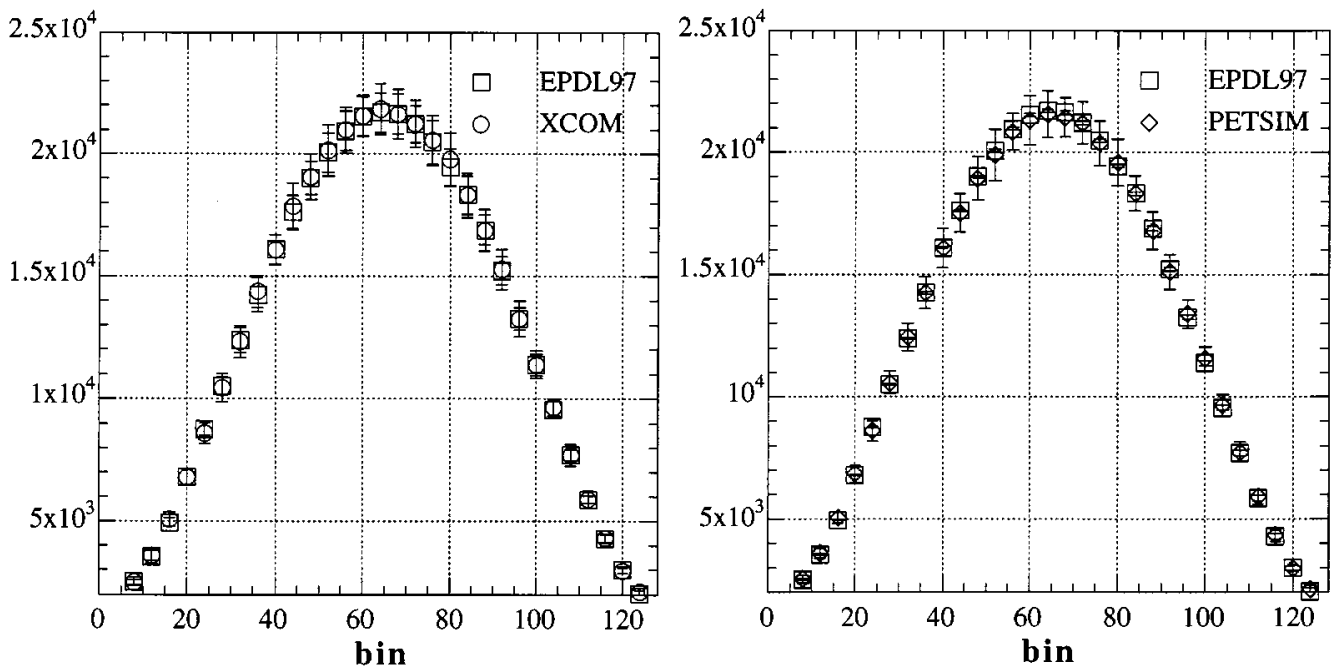

(b)

Fig. 7. Comparison between simulated scatter profiles of a $20 \mathrm{~cm}$ diameter uniformly water-filled right circular cylinder generated using the different photon cross-section libraries: (a) $380 \mathrm{keV}$ LLD and (b) $450 \mathrm{keV}$ LLD. Scatter profiles are summed over all angles and error bars shown on the plots.

[16] are public domain packages. The first version of SIMSET used PETSIM's parametric model then it was modified later on to incorporate more recent data from LLNL (EPDL94) [18]. Hence, the results obtained with PETSIM can be extrapolated to the first version of SIMSET and those obtained with EPDL97 to the current version since for the energies of interest in this paper, there is no significant difference between EPDL94 and EPDL97.

In the last few years, the LLNL photon and electron data bases have been greatly improved in terms of the detail included as well as the accuracy of the data. At the same time, there has been an enormous increase in available inexpensive computer power. 
TABLE IV

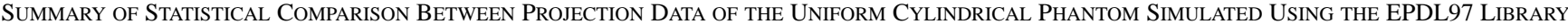

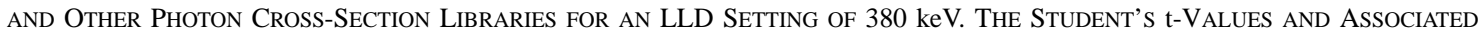
Statistical Difference Are Shown. The Critical t-Value $\left(t_{\text {crit }}=1.645\right)$ is Related to a Probability of 0.05 FOR the Null Hypothesis to OCcur (“The Two Distributions ARE Not the SAME”)

\begin{tabular}{lcc}
\hline Photon cross section library & Student's t-value & Probability \\
\hline GEANT & 32.481 & $<0.025$ \\
XCOM & 2.465 & $<0.025$ \\
PHOTX & 22.907 & $<0.025$ \\
PETSIM & 29.511 & $<0.025$ \\
\hline
\end{tabular}
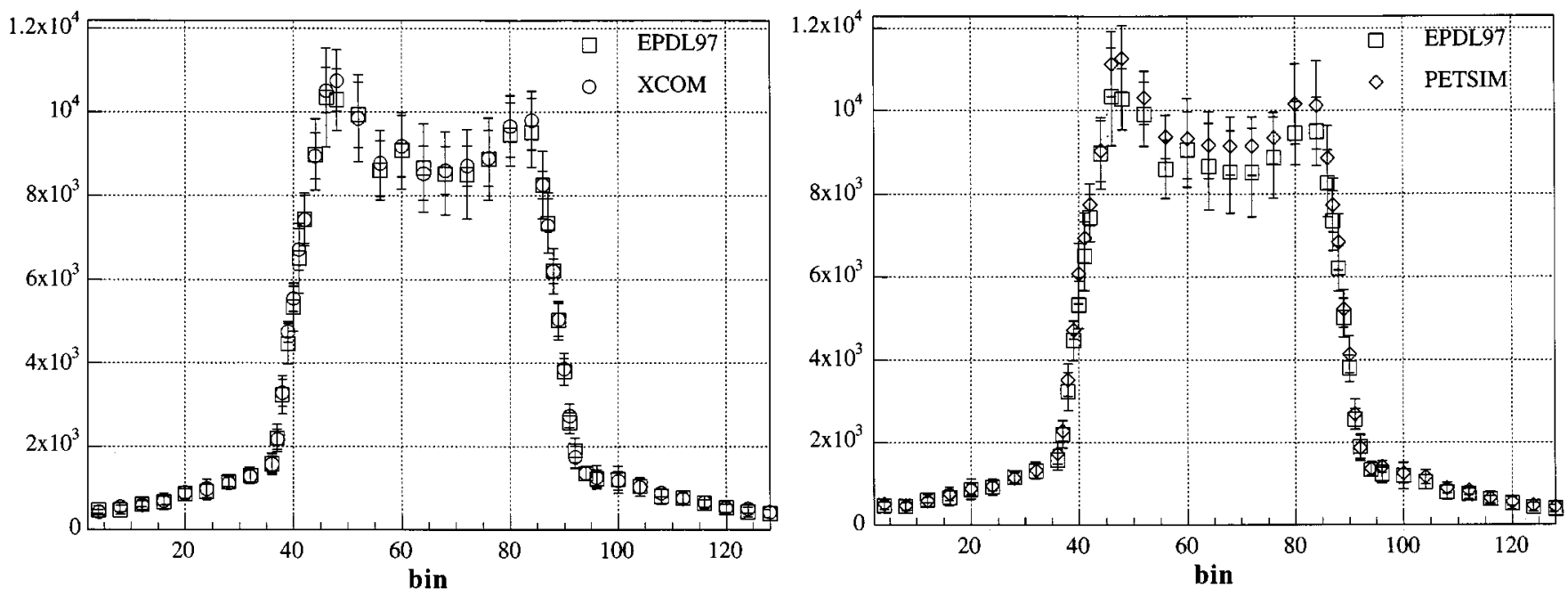

(a)
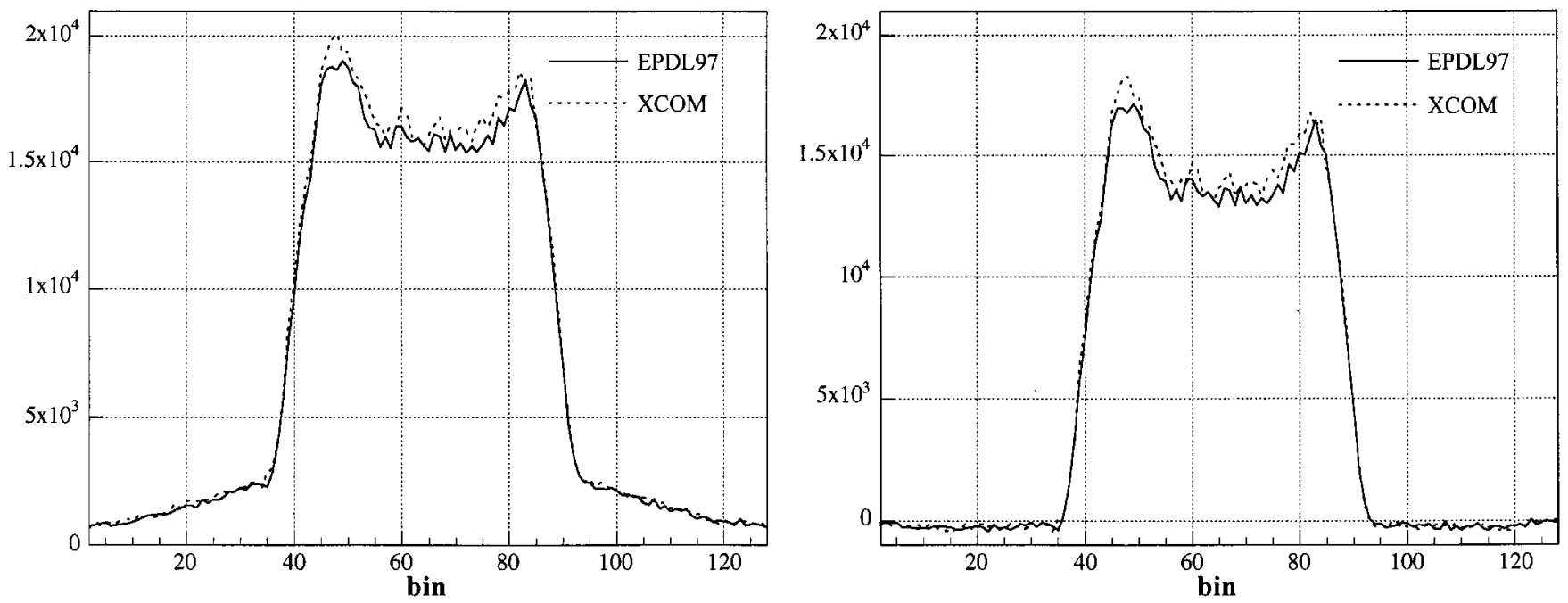

(b)

Fig. 8. (a) Integral profile through a 2-D projection (single angular view) of a simulated Hoffman brain phantom generated using the different photon cross-section libraries. (b) Same as (a) for EPDL97 and XCOM before (left) and after (right) applying scatter correction to the sum of the ten batch realizations of Monte Carlo simulations. Error bars are shown on the plots.

The combination of improved photon and electron databases and increased availability of inexpensive computer power allows us today to calculate results in greater detail, with greater accuracy, using accurate methods such as Monte Carlo modeling. The cross-section values produced by the LLNL in collaboration with NIST are thought to be the most up-to-date and accurate coefficients available, and the LLNL database is now a national and international standard [25]. EPDL97 is already a standard in the nuclear reactor industry, since its adoption by the Cross Section Evaluation Working Group (CSEWG) for use with ENDF/B-VI. In the author's opinion, it would be desirable that EPDL97 is adopted as an International standard not because it is perfect, but rather because it could serve as a standard against which everyone can test, and based on user feed- 


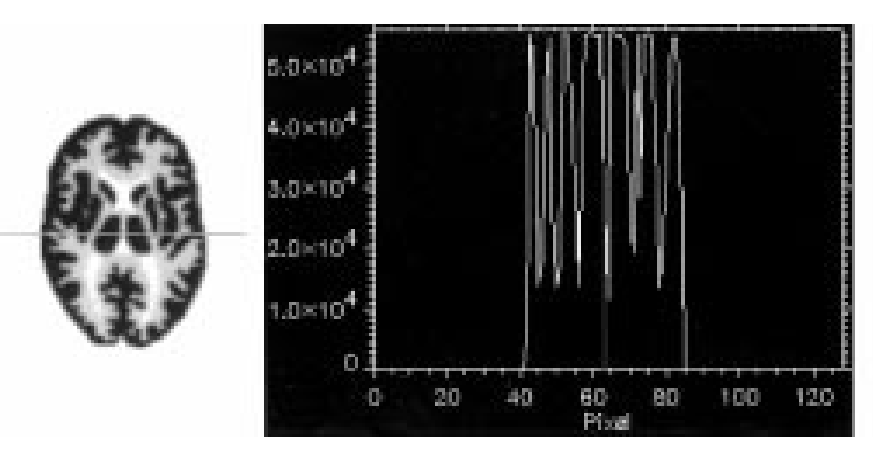

(a)

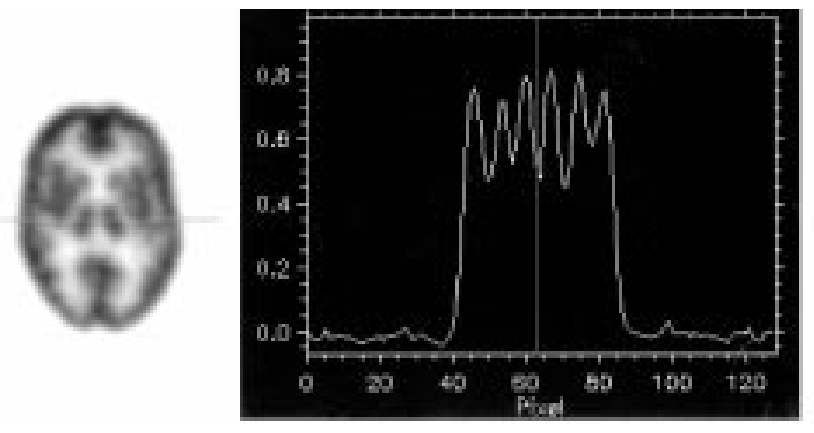

(c)

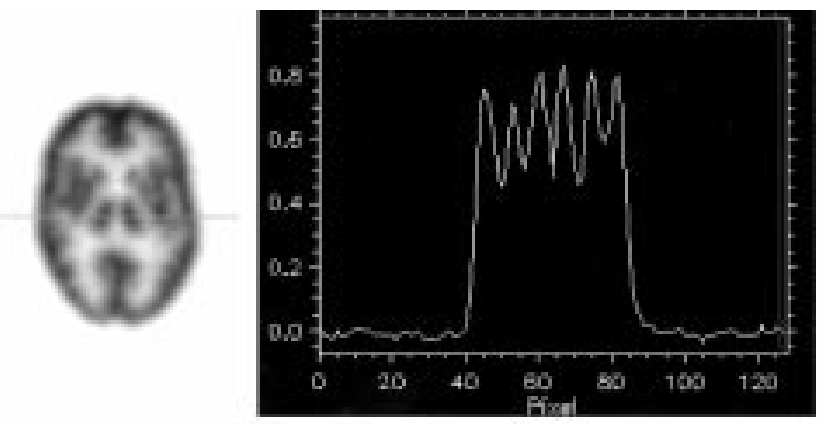

(e)
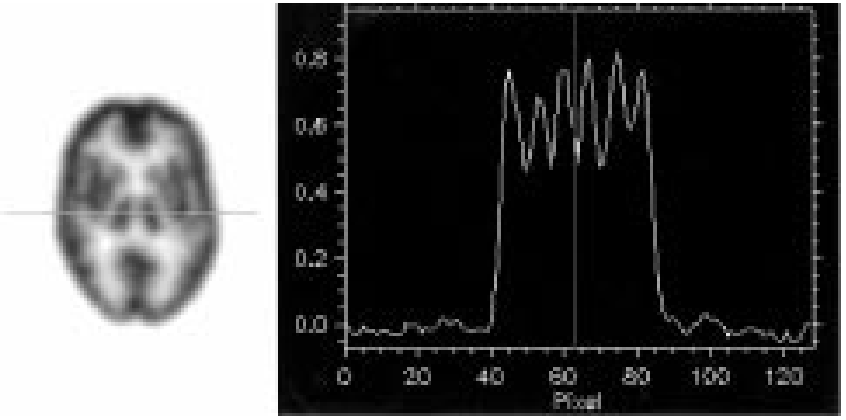

(b)

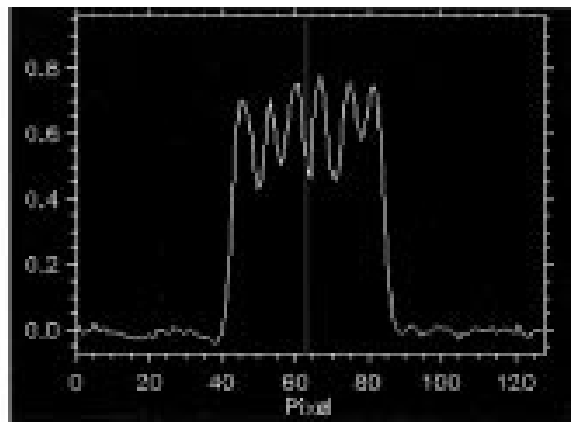

(d)

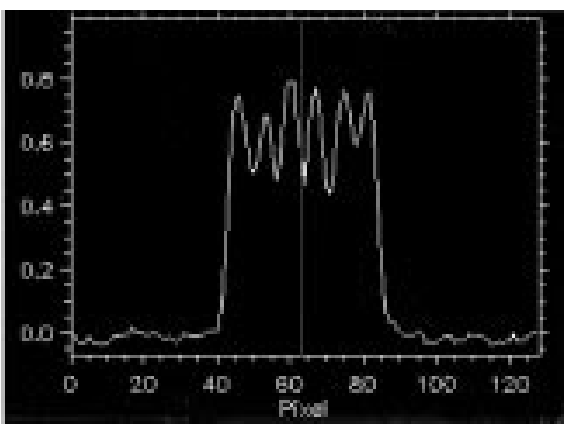

(f)

Fig. 9. Reconstructed images of the 3-D Hoffman brain phantom and horizontal profiles for scatter corrected data sets generated with different photon cross-section libraries: (a) The 3-D reference image, (b) EPDL97, (c) GEANT, (d) XCOM, (e) PHOTX, (f) PETSIM.

back the authors of this library would then maintain EPDL97 up-to-date to reflect the latest measurements and calculations. Therefore, we highly recommend that EPDL97 cross-section data serve as a standard and supersede earlier cross-section libraries used in Monte Carlo codes to simulate medical imaging systems. This will help to eliminate potential differences between the results obtained with different codes. The results presented in this paper have shown that the effect of using different photon cross-section libraries is noticeable on both generated projection data and reconstructed images (Figs. 6-9). The peaks are not of equal heights and the valleys not of equal deep in most images especially Fig. 9(f), while Fig. 9(d) has peaks that are more equal in height. The data sets were obtained with the same statistics and random number seeds, so the differences can only be attributed to the cross-section libraries used to generate them. The EPDL97 library was carefully designed in the form of look-up tables for efficiency in data storage, access, and use by the Monte Carlo software [37]. This work presented just a direct comparison of the photon interaction data. It doesn't address the companion Evaluated Electron Data Library (EEDL), and
Evaluated Atomic Data Library (EADL), which are needed to complete the picture of photon transport and energy deposition. Those libraries are the only complete and consistent set of data libraries available to treat the complete coupled photon-electron transport problem. These libraries include details that were not previously available, or not considered when performing calculations using traditional photon interaction data.

\section{ACKNOWLEDGMENT}

The author gratefully thanks the anonymous reviewers for their useful comments. This paper is dedicated to the memory of my beloved father.

\section{REFERENCES}

[1] H. Zaidi, "Quantitative SPECT: Recent developments in detector response, attenuation and scatter correction techniques," Phys. Med., vol. 12, no. 2, pp. 101-117, 1996.

[2] - "Relevance of accurate Monte Carlo modeling in nuclear medical imaging," Med. Phys., vol. 26, no. 4, pp. 574-608, 1999. 
[3] J. H. Hubbell, "Review of photon interaction cross section data in the medical and biological context," Phys. Med. Biol., vol. 44, no. 1, pp. $1-22,1999$.

[4] Lawrence Livermore National Laboratory, Livermore, CA, UCRL-50400, Rev. 5, vol. 6, 1997.

[5] M. J. Berger and J. H. Hubbell, XCOM: Photon cross sections on a personal computer NBSIR 87-3597, , 1987.

[6] Y. Sakamoto, "Photon cross section data PHOTX for PEGS4 code," in Proc. Third EGS4 Users' Meeting Japan, 1993, pp. 77-82.

[7] CERN Program Library, Geneva, Switzerland, 1994.

[8] Y. Picard, C. J. Thompson, and S. Marrett, "Improving the precision and accuracy of Monte Carlo simulation in positron emission tomography," IEEE Trans. Nucl. Sci., vol. 39, pp. 1111-1116, 1993.

[9] H. Zaidi, A. H. Scheurer, and C. Morel, "An object-oriented Monte Carlo simulator for 3-D positron tomographs," Comput. Methods Programs Biomed., vol. 58, no. 2, pp. 133-145, 1999.

[10] M. Ljungberg, S.-E. Strand, and M. A. King, Monte Carlo Calculations in Nuclear Medicine: Applications in Diagnostic Imaging. London, U.K.: IOP, 1998.

[11] "The EGS4 Code System SLAC-256," Stanford Linear Accelerator Center, Stanford, CA, 1985.

[12] M. Ljungberg and S.-E. Strand, "A Monte Carlo program for the simulation of scintillation camera characteristics," Comput. Methods Programs Biomed., vol. 29, no. 4, pp. 257-272, 1989.

[13] J. C. Yanch and A. B. Dobrzeniecki, "Monte Carlo simulation in SPECT: Complete 3-D modeling of source, collimator and tomographic data acquisition," IEEE Trans. Nucl. Sci., vol. 40, pp. 198-203, 1993.

[14] M. F. Smith, C. E. Floyd, and R. J. Jaszczak, "A vectorized Monte Carlo code for modeling photon transport in SPECT," Med. Phys., vol. 20, no. 4, pp. 1121-1127, 1993.

[15] C. J. Thompson, J.-M. Cantu, and Y. Picard, "PETSIM: Monte Carlo program simulation of all sensitivity and resolution parameters of cylindrical positron imaging system," Phys. Med. Biol., vol. 37, no. 3, pp. 731-749, 1992

[16] R. L. Harrison, S. D. Vannoy, D. R. Haynor, S. B. Gillispie, M. S. Kaplan, and T. K. Lewellen, "Preliminary experience with the photon history generator module for a public-domain simulation system for emission tomography in," in Proc. IEEE Medical Imaging Conf., San Francisco, CA, 1994, pp. 1154-1158.

[17] H. Zaidi, C. Labbé, and C. Morel, "Implementation of an environment for Monte Carlo simulation of fully 3-D positron tomography on a highperformance parallel platform," Parallel Comput., vol. 24, no. 9-10, pp. 1523-1536, 1998

[18] M. S. Kaplan, R. L. Harrison, and S. D. Vannoy, "Coherent scatter implementation for SimSET," IEEE Trans. Nucl. Sci., vol. 45, no. 3, pp. 3064-3068, 1998

[19] E. Storm and H. I. Israel, "Photon cross sections from $1 \mathrm{keV}$ to $100 \mathrm{MeV}$ for elements Z = 1 to Z = 100," Nucl. Data Tables, vol. A7, pp. 565-681, 1970.

[20] Los Alamos National Laboratory, Los Alamos, NM, LA-12 625-M, 1977.

[21] J. H. Hubbell, W. J. Veigele, E. A. Briggs, R. T. Brown, D. T. Cromer, and R. J. Howerton, "Atomic form factors, incoherent scattering functions, and photon scattering cross sections," J. Phys. Chem. Ref., pp. 471-538, 1975.

[22] Lawrence Livermore National Laboratory, Livermore, CA, Rev. 4, Part A: $Z=1$ to 50 and Part $B: Z=51$ to 100 , vol. 6,1989 .

[23] D. K. Trubey, M. J. Berger, and J. H. Hubbell, "Photon cross sections for ENDF/B-IV," in Proc. American Nuclear Society Topical Meeting, Advancement Nuclear Engineering Computing Radiation Shielding, Santa Fe, NM, Apr. 9-13, 1989, p. 1989.
[24] J. A. Halbleib, R. P. Kensek, G. D. Valdez, S. M. Seltzer, and M. J Berger, "ITS: The integrated TIGER series of electron/photon transport codes-version 3.0," IEEE Trans. Nucl. Sci., vol. 39, pp. 1025-1030, 1992.

[25] J. M. Boone and A. E. Chavez, "Comparison of X-ray cross sections for diagnostic and therapeutic medical physics," Med. Phys., vol. 23, no. 12, pp. 1997-2005, 1996

[26] "International Commission on Radiological Units and Measurements Report,” ICRU, Bethesda, MD, vol. 44, 1989.

[27] O. Klein and Y. Nishina, "Über die Streuung von Strahlung durch freie Elektronen nach der neuen relativistischen Quantendynamik von Dirac," Z. Phys., vol. 52, pp. 853-868, 1929.

[28] E. J. Hoffman, P. D. Cutler, W. M. Digby, and J. C. Mazziotta, "3-D phantom to simulate cerebral blood flow and metabolic images for PET," IEEE Trans. Nucl. Sci., vol. 37, no. 2, pp. 616-620, 1990.

[29] T. J. Spinks, T. Jones, D. L. Bailey, D. W. Townsend, S. Grootoonk, P. M. Bloomfield, M.-C. Gilardi, M. E. Casey, B. Sipe, and J. Reed, "Physical performance of a positron tomograph for brain imaging with retractable septa," Phys. Med. Biol., vol. 37, no. 8, pp. 1637-1655, 1992.

[30] Statsoft, Inc.. Electronic Statistics Textbook [Online]. Available: http://www.statsoft.com/textbook/stathome.html

[31] D. L. Bailey and S. R. Meikle, "A convolution-subtraction scatter correction method for 3-D PET," Phys. Med. Biol., vol. 39, pp. 411-424, 1994.

[32] H. Zaidi. Statistical reconstruction-based scatter correction: A new method for 3-D PET. presented at Conf. Proc. World Congr. Med. Phys. Biomed. Engi. 2000. [Online]. Available: http://dmnu-pet5.hcuge.ch/

[33] P. E. Kinahan and J. G. Rogers, "Analytic 3-D image reconstruction using all detected events," IEEE Trans. Nucl. Sci., vol. 36, pp. 964-968, 1989.

[34] C. Michel, A. Bol, T. Spinks, D. W. Townsend, D. Bailey, S. Grootoonk, and T. Jones, "Assessment of response function in two PET scanners with and without interplane septa," IEEE Trans. Med. Imag., vol. 10, no. 3, pp. 240-248, 1991

[35] L. E. Adam and C. C. Watson, "Experimental determination of the lower energy discriminator level for a positron tomograph," Nuklearmedizin, vol. 38, pp. 61-65, 1999.

[36] D. Vozza, C. Moisan, and S. Paquet, "An improved model for energy resolution of multicrystal encoding detectors for PET," IEEE Trans. Nucl. Sci., vol. 44, pp. 179-183, 1997.

[37] H. Zaidi, C. Labbé, and C. Morel, "Improvement of the performance and accuracy of PET Monte Carlo simulations," in Proc. SPIE Int. Symp. Medical Imaging, vol. 3659, J. M. Boone and J. T. Dobbins, Eds., San Diego, CA, 1999, pp. 137-140.

[38] Lawrence Livermore National Laboratory, Livermore, CA, UCRL-ID $117796,1994$.

[39] J. H. Hubbell, "Photon cross sections, attenuation coefficients and energy absorption coefficients from $10 \mathrm{keV}$ to $100 \mathrm{GeV}$ National Bureau of Standards (US) Report,", 1969

[40] G. Tsang, C. Moisan, and J. G. Rogers, "A simulation to model position encoding multicrystal PET detectors," IEEE Trans Nucl. Sci., vol. 42, no. 6, pp. 2236-2243, 1995.

[41] J. H. Hubbell, W. J. Veigele, E. A. Briggs, R. T. Brown, D. T. Cromer, and R. J. Howerton, "Atomic form factors, incoherent scattering functions, and photon scattering cross sections," J. Phys. Chem. Ref., vol. 6, pp. 615-616, 1977. 